\title{
Investigating the biological properties of carbohydrate derived fulvic acid (CHD-FA) as a potential novel therapy for the management of oral biofilm infections
}

\author{
Leighann Sherry, Emma Millhouse, David F Lappin, Colin Murray, Shauna Culshaw, Christopher J Nile \\ and Gordon Ramage*
}

\begin{abstract}
Background: A number of oral diseases, including periodontitis, derive from microbial biofilms and are associated with increased antimicrobial resistance. Despite the widespread use of mouthwashes being used as adjunctive measures to control these biofilms, their prolonged use is not recommended due to various side effects. Therefore, alternative broad-spectrum antimicrobials that minimise these effects are highly sought after. Carbohydrate derived fulvic acid (CHD-FA) is an organic acid which has previously demonstrated to be microbiocidal against Candida albicans biofilms, therefore, the aims of this study were to evaluate the antibacterial activity of CHD-FA against orally derived biofilms and to investigate adjunctive biological effects.
\end{abstract}

Methods: Minimum inhibitory concentrations were evaluated for CHD-FA and chlorhexidine (CHX) against a range of oral bacteria using standardised microdilution testing for planktonic and sessile. Scanning electron microscopy was also employed to visualise changes in oral biofilms after antimicrobial treatment. Cytotoxicity of these compounds was assessed against oral epithelial cells, and the effect of CHD-FA on host inflammatory markers was assessed by measuring mRNA and protein expression.

Results: CHD-FA was highly active against all of the oral bacteria tested, including Porphyromonas gingivalis, with a sessile minimum inhibitory concentration of $0.5 \%$. This concentration was shown to kill multi-species biofilms by approximately 90\%, levels comparable to that of chlorhexidine (CHX). In a mammalian cell culture model, pretreatment of epithelial cells with buffered CHD-FA was shown to significantly down-regulate key inflammatory mediators, including interleukin-8 (IL-8), after stimulation with a multi-species biofilm.

Conclusions: Overall, CHD-FA was shown to possess broad-spectrum antibacterial activity, with a supplementary function of being able to down-regulate inflammation. These properties offer an attractive spectrum of function from a naturally derived compound, which could be used as an alternative topical treatment strategy for oral biofilm diseases. Further studies in vitro and in vivo are required to determine the precise mechanism by which CHD-FA modulates the host immune response.

Keywords: Fulvic acid, Chlorhexidine, Biofilm, Antibacterial, Periodontitis, Inflammation

\footnotetext{
* Correspondence: gordon.ramage@glasgow.ac.uk

Infection and Immunity Research Group, Glasgow Dental School, School of

Medicine, College of Medical, Veterinary and Life Sciences, University of

Glasgow, 378 Sauchiehall Street, Glasgow G2 3JZ, UK
} 


\section{Background}

Dental caries, gingivitis and periodontitis are the most common microbial diseases of the oral cavity, with the majority associated with a polymicrobial biofilm $[1,2]$. Biofilms are a collection of multicellular microorganisms attached to one another or upon a surface, and are embedded by a protective layer of extracellular matrix (ECM) [3]. Biofilms are of greater clinical importance than their free-floating planktonic counterparts because of their innate ability to resist antimicrobial therapy and host defences. This is due to the extensive ECM production and other factors such as increased extrusion of antimicrobials through enhanced efflux pump activity $[3,4]$.

Antimicrobial mouthwashes are one of the main therapeutic and preventative strategies currently used in the management of oral biofilm diseases, of which chlorhexidine $(\mathrm{CHX})$ is widely accepted as the 'gold standard' [5]. This antiseptic agent has superior activity to its comparators, and is both cidal and static against microorganisms present in oral biofilms with roles in the pathogenesis of oral disease. Moreover, its substantivity provides prolonged activity through its ability to adsorb onto the pellicle found on enamel surfaces of teeth [6]. Despite this, various studies have shown long-term use of CHX may not be practical as it is associated with staining of the teeth and taste alterations $[7,8]$. Furthermore, recent reports of adverse events, including anaphylactic reactions, to this compound have been described [9]. It has also been shown recently to be ineffective against biofilms grown from clinical isolates [10].

The prevention and treatment of oral biofilm diseases, such as periodontal diseases and mucosal infections, may benefit from a compound that has the potency of CHX with minimal side effects, but also elicits adjunctive biological properties, such as alteration of inflammatory pathways, which are clearly important in the pathogenesis of oral biofilm disease [11,12]. A previous study has shown $\mathrm{CHX}$ is able to down-regulate inflammatory mediators when challenged with a bacterial stimuli [13], though toxicological aspects of $\mathrm{CHX}$ may be the reason for the decreased expression. We have also shown the benefit of using natural agents in the management of oral infections, where tea tree oil (TTO) was not only nontoxic, but was able to dampen the host immune response to a fungal stimulus [14]. Furthermore, our group have previously assessed the antiseptic activity of carbohydratederived fulvic acid (CHD-FA), where it was shown that the compound was equally effective against Candida albicans planktonic and biofilm cells. Mechanistically this was identified as a membrane disruption process that was not impacted by defined biofilm adaptive resistance mechanisms [15]. CHD-FA is a colloidal organic acid, which is a major constituent of humic acids. A purified form of CHD-FA has recently been produced by a patented process, which has been shown to be non-toxic in a rat wound model, with suggestions of anti-inflammatory activity [16]. Moreover, a recent randomized, double blind, controlled trial indicated that it was well-tolerated in a clinical study of eczema [17].

The purpose of this study was to investigate whether CHD-FA has a broad-spectrum of activity against microbial biofilms of oral relevance to determine whether it could be used as an alternative to CHX based mouthwashes, which have known side effects from prolonged use. The secondary aim of the study was to determine whether the antibacterial concentration of CHD-FA had any adjunctive immunomodulatory properties, as reported elsewhere [16]. We report that CHD-FA displays rapid microbiocidal activity against orally relevant biofilms, and that it is also able to down-regulate the expression of proinflammatory molecules in orally relevant epithelial cells.

\section{Methods}

\section{Culture conditions and standardisation}

A selection of laboratory strains of commensal and pathogenic bacteria associated with oral biofilms disease were used in this study, including Porphyromonas gingivalis ATCC 33277 and Fusobacterium nucleatum ATCC 10596, which were maintained at $37^{\circ} \mathrm{C}$ on fastidious anaerobic agar (FAA [Lab M, Lancashire, UK]) under anaerobic conditions $\left(85 \% \mathrm{~N}_{2}, 10 \% \mathrm{CO}_{2}\right.$ and $5 \% \mathrm{H}_{2}$, [Don Whitley Scientific Limited, Shipley, UK]). Streptococcus mutans 10449, Streptococcus mitis NCTC 12261, Aggregatibacter actinomycetemcomitans OSM 1123 and Enterococcus faecalis NCTC 5957 were grown and maintained at $37^{\circ} \mathrm{C}$ on Colombia blood agar (CBA [Oxoid, Hampshire, UK] in $5 \% \mathrm{CO}_{2}$. All isolates were stored indefinitely in Microbank ${ }^{\odot}$ vials (Pro-Lab Diagnostics, Cheshire, UK) at $-80^{\circ} \mathrm{C}$.

$P$. gingivalis and F. nucleatum were propagated in $10 \mathrm{ml}$ Schaedler's anaerobic broth (Oxoid), S. mitis and A. actinomycetemcomitans were grown in $10 \mathrm{ml}$ Tryptic Soy Broth (TSB [Sigma-Aldrich, Dorset, UK]) supplemented with $0.6 \%$ yeast extract and $0.8 \%$ glucose. E. faecalis was grown in TSB with $0.25 \%$ glucose, and $S$. mutans was grown in $10 \mathrm{ml}$ brain heart infusion (BHI [Sigma-Aldrich]), all at $37^{\circ} \mathrm{C}$ and at appropriate atmospheric conditions. Overnight cultures were washed by centrifugation (1000 xg) and resuspended in $10 \mathrm{ml}$ PBS. All bacteria were then standardised and adjusted to a final working concentration of $5 \times 10^{4}$ and $1 \times 10^{7}$ cells $/ \mathrm{ml}$ for planktonic and sessile susceptibility testing, respectively.

\section{Antibacterial susceptibility testing of planktonic and biofilm cells}

During the course of this study two active compounds from the oral hygiene products Dentracine (Fulhold Ltd, Cape Town, South Africa) and Corsodyl (GlaxoSmithKline 
Consumer Health Care, UK) were tested, namely CHD-FA and $\mathrm{CHX}$, respectively.

Antimicrobial testing to determine minimum inhibitory concentrations (MICs) of planktonic cells (PMIC) was performed using the CLSI M11-A8 broth microdilution method for anaerobic bacteria [18] and CLSI M7-A9 for bacteria grown in 5\% $\mathrm{CO}_{2}$ [19]. Minimum bactericidal concentrations (MBC) were also determined by standard plating methods.

For biofilm testing standardised P. gingivalis, $F$. nucleatum, $S$. mitis and A. actinomycetemcomitans were grown for $72 \mathrm{~h}$ and $E$. faecalis for $24 \mathrm{~h}$ in their respective media and atmospheric conditions, with the exception of $S$. mutans which was grown in BHI supplemented with $2 \%$ sucrose for $48 \mathrm{~h}$. Biofilms were grown statically in commercially available 96-well flat bottomed microtitre plates (Corning Incorporated, NY, USA) and sessile susceptibility testing was performed as described elsewhere [20]. Following antimicrobial treatment, biofilms were washed twice with PBS and 10\% alamarBlue $^{\ominus}$ (Invitrogen, Paisley, UK) was added to the biofilms prior to incubation for $4 \mathrm{~h}$ in the dark [21]. Sessile minimum inhibitory concentrations (SMICs) were read visually and no change in colour was defined as the SMIC. Testing of all planktonic and sessile isolates was performed in quadruplicate on two separate occasions.

\section{Antibacterial susceptibility testing of a multi-species periodontal biofilm}

A multi-species periodontal biofilm model consisting of $P$. gingivalis, F. nucleatum, $S$. mitis and $A$. actinomycetemcomitans was developed for antimicrobial testing. All bacterial species were standardised to $1 \times 10^{7} \mathrm{cfu} / \mathrm{mL}$ in artificial saliva (AS) as previously described [22]. This was comprised of porcine stomach mucins $(0.25 \% \mathrm{w} / \mathrm{v})$, sodium chloride $(0.35 \mathrm{w} / \mathrm{v})$, potassium chloride $(0.02 \mathrm{w} / \mathrm{v})$, calcium chloride dihydrate $(0.02 \mathrm{w} / \mathrm{v})$, yeast extract $(0.2 \mathrm{w} / \mathrm{v})$, lab lemco powder $(0.1 \mathrm{w} / \mathrm{v})$, proteose peptone $(0.5 \mathrm{w} / \mathrm{v})$ in $\mathrm{ddH}_{2} \mathrm{O}$. Urea was diluted in PBS (40\% w/v) and added to a final concentration of $0.05 \%(\mathrm{v} / \mathrm{v})$ in AS. Biofilms were prepared in 24 well plates (Corning, NY, USA) containing customised Thermanox $^{\mathrm{TM}}$ coverslips (13 $\mathrm{mm}$ diameter, Fisher Scientific). For the addition of each bacterial species to the biofilm a standardised bacterial suspension was prepared in $500 \mu \mathrm{L}$ of AS. Initially, S. mitis biofilms were grown for $24 \mathrm{~h}$. Media was then removed and standardised F. nucleatum added, which was incubated anaerobically for a further $24 \mathrm{~h}$. The supernatant was again removed and standardised $P$. gingivalis and $A$. actinomycetemcomitans in AS added to the biofilm. This was then incubated at $37^{\circ} \mathrm{C}$ in an anaerobic chamber for a further 4 days; each day supernatants were replaced with fresh AS. As CHD-FA was shown to be active at $0.5 \% \mathrm{v} / \mathrm{v}$ against all bacterial biofilms tested in this study, this concentration was used in addition to $0.2 \% \mathrm{v} / \mathrm{v} \mathrm{CHX}$ to treat multispecies biofilms for $30 \mathrm{~min}$, before carefully washed with PBS, and biofilm viability determined using alamarBlue ${ }^{\ominus}$. The absorbance was read at $570 \mathrm{~nm}$ and the reference wavelength at $600 \mathrm{~nm}$. The percentage reduction in biofilm viability was calculated according to the manufacturer's instructions. This study was performed on three separate occasions in triplicate.

Following the antimicrobial treatment, biofilms were retained and used to quantify the number of each bacterial species found after CHD-FA and CHX treatment compared to the untreated control. Briefly, biofilms were sonicated in $1 \mathrm{~mL}$ of PBS for $10 \mathrm{~min}$ and DNA extracted using the MasterPure Gram Positive DNA Purificiation Kit (Epicentre ${ }^{\oplus}$, Cambridge, UK), following manufacturers instructions. $1 \mu \mathrm{L}$ of extracted DNA was added to a mastermix containing $12.5 \mu \mathrm{L}$ SYBR $^{\bullet}$ GreenER $^{\mathrm{TM}}, 9.5 \mu \mathrm{L}$ UV-treated RNase-free water and $1 \mu \mathrm{L}$ of $10 \mu \mathrm{M}$ forward/ reverse primers for each bacterial species. The primers used were as follows:

A. actinomycetemcomitans F - 5'GAACCTTACCT ACTCTTGACATCCGAA3', A. actinomycetemcomitans R - 5'TGCAGCACCTGTCTCAAAGC3', F. nucleatum F - 5'GGATTTATTGGGCGTAAAGC3', F. nucleatum R - 5'GGCATTCCTACAAATATCTACGAA3', P. gingivalis $\mathrm{F}$ - 5'GCGCTCAACGTTCAGCC3', P. gingivalis $\mathrm{R}$ - 5'CACGAATTCGCCTGC3', S. mitis F - 5'GATA CATAGCCGACCTGAG3', S. mitis R - 5'CCATTGCC GAAGATTCC3'.

Three independent replicates from each parameter were analysed in triplicate using MxProP Quantitative PCR machine and MxProP 3000 software (Stratagene, Amsterdam, Netherlands). Samples were quantified based upon a previously established standard curve made up of known bacterial counts.

\section{Ultrastructural changes of bacterial biofilms}

Scanning electron microscopy (SEM) was performed on S. mutans, E. faecalis, and the multispecies biofilms. Cells were standardised in appropriate media, as described above, and grown directly onto Thermanox ${ }^{\mathrm{Tm}}$ coverslips (Nunc, Roskilde, Denmark) to allow biofilm formation. Following maturation biofilms were carefully washed with PBS before their respective treatments. Biofilms were then carefully washed twice with PBS and then fixed in $2 \%$ para-formaldehyde, $2 \%$ gluteraldehyde and $0.15 \mathrm{M}$ sodium cacodylate, and 0.15\% w/v Alcian Blue, $\mathrm{pH} 7.4$, and prepared for SEM as previously described [23]. The specimens were sputter-coated with gold and viewed under a JEOL JSM-6400 scanning electron microscope. Images were assembled using Photoshop software (Adobe, San Jose, CA, USA). 


\section{Toxicity of CHD-FA upon an oral epithelial cell line}

OKF6/TERT2 cells (gifted by the Rheinwald laboratory, Brigham and Woman's Hospital, Boston, USA), an immortalised human oral keratinocyte cell line, were used for determining the cytotoxicity of CHD-FA. Cells were grown to $90 \%$ confluence in keratinocyte serum-free medium (KSFM) at $37^{\circ} \mathrm{C}$ in $5 \% \mathrm{CO}_{2}$ and seeded at a density of $1 \times 10^{5}$ cells $/ \mathrm{ml}$ in a 24 well plate. Once the cells reached $80-90 \%$ confluence, the cells were carefully washed with PBS before treatment with $0.5 \%$ (v/v) CHDFA at the native $\mathrm{pH} 2.0$ and a neutral $\mathrm{pH}$ of 7.0 and $0.2 \%$ (v/v) CHX for $30 \mathrm{~min}$. After $30 \mathrm{~min}$, the compounds were removed and the cells carefully washed with PBS to remove any residual actives. Cells were incubated in KSFM for 4 and $24 \mathrm{~h}$ before cellular viability was assessed using the alamarBlue ${ }^{\oplus}$ assay, as described above. Viability studies were carried out in triplicate, on three separate occasions.

\section{Assessing immunomodulatory properties of CHD-FA}

OKF6/TERT2 cells were grown to 90\% confluence in 24 well plates in defined-KSFM then pre-treated with $0.5 \%$ CHD-FA ( $\mathrm{pH} \mathrm{7.0)} \mathrm{for} 30 \mathrm{~min}$. CHD-FA at $\mathrm{pH} 2.0$ was toxic against the cell line used in this study and therefore could not allow us to analyse any potential immunomodulatory properties of this compound. Therefore, CHD-FA buffered to $\mathrm{pH} 7.0$ was used to assess any further biological properties of the compound. 0.5\% CHD-FA at $\mathrm{pH} 2.0$ and $0.2 \% \mathrm{CHX}$ were shown to be toxic to epithelial cells, so were not further investigated. Cells were washed with PBS to remove residual CHD-FA. As an inflammatory agonist we used the multispecies periodontal biofilm, as described above, which was attached to the underside of a hanging cell culture insert (Millipore, Massachusetts, USA) using Vaseline ${ }^{\oplus}$, then laid adjacent to the cell monolayer. The cells were incubated with the periodontal biofilm for 4 and $24 \mathrm{~h}$ at $37^{\circ} \mathrm{C}$ in $5 \% \mathrm{CO}_{2}$. Cells not pretreated with CHD-FA, or not challenged with biofilms, served as appropriate controls. Following stimulation, supernatants and cell lysates were retained to assess the regulation of a panel of pro-inflammatory mediators.

Initial gene expression analysis was carried out using a custom designed $\mathrm{RT}^{2}$ Profiler PCR Array (Qiagen, Crawley, UK). $\mathrm{RT}^{2}$ Profiler arrays are a $\mathrm{SYBR}^{\odot}$ GreenER $^{\mathrm{Ts}}$ based real-time PCR that allow for the detection of several genes of interest, simultaneously. Briefly, $24 \mu \mathrm{l}$ of a mastermix containing $\mathrm{SYBR}^{\odot}$ GreenER ${ }^{\mathrm{T}}$, cDNA synthesised using the $\mathrm{RT}^{2}$ First Strand kit (Qiagen) and RNasefree water was added to each well of the $\mathrm{RT}^{2}$ Profiler plate, which already contained the forward and reverse primers for the genes of interest (IL-1 $\alpha$, IL-1 $\beta$, IL-6, TNF, CSF2, CSF3, IL-8, CXCL1, CXCL3, CXCL5, CCL1 and GAPDH). Two replicates of each condition were used in the $\mathrm{RT}^{2}$ Profiler, which was carried out on two separate occasions.
IL-8 gene expression was analysed using SYBR ${ }^{\oplus}$ Green based qPCR (Invitrogen), using GAPDH as a housekeeping gene. The primers used were as follows: IL-8 F 5' CAGAGACAGCAGAGCACACAA3', IL-8 R 5'TTAGC ACTCCTTGGCAAAAC3', GAPDH F 5'CAAGGCTGA GAACGGGAAG3', GAPDH R 5'GGTGGTGAAGACG CCAGT3'. Briefly, RNA was extracted from cell lysates (Qiagen, Crawley, UK) and $55 \mathrm{ng} / \mu \mathrm{l}$ of cDNA synthesised using the $\mathrm{RT}^{2}$ First Strand CDNA synthesis kit (Qiagen, Crawley, UK), as per manufacturers instructions. $1 \mu \mathrm{l}$ of synthesised cDNA was added to a mastermix containing $12.5 \mu \mathrm{l} \mathrm{SYBR}{ }^{\odot}$ GreenER $^{\mathrm{TM}}, 10.5 \mu \mathrm{l}$ UVtreated RNase-free water and $0.5 \mu \mathrm{l}$ of forward/reverse primers. Three independent replicates from each parameter were analysed in duplicate using MxProP Quantitative PCR machine and MxProP 3000 software (Stratagene, Amsterdam, Netherlands) and gene expression normalised to the housekeeping gene GAPDH according to the $2^{-\Delta \Delta C T}$ method [24].

Interleukin 8 (IL-8) release into cell culture supernatants was assessed by ELISA (Invitrogen, Paisley, UK), as per manufacturer's instructions. Results were calculated using a 4-parameter curve fit, quantifying colometric changes at 630 nm (BMG-Labtech, Ortenberg, Germany).

\section{Statistical analysis}

Graph production, data distribution and statistical analysis were performed using GraphPad Prism (version 4; La Jolla, CA, USA). After assessing whether data conformed to a normal distribution by before and after data transforms, One-way Analysis of Variance (ANOVA) and $t$ tests were used to investigate significant differences between independent groups of data that approximated to a Gaussian distribution. A Bonferroni correction was applied to the $\mathrm{p}$ value to account for multiple comparisons of the data. Non-parametric data was analysed using the Mann-Whitney $U$-test to assess differences between two independent sample groups. Student t-tests were used to measure statistical differences between the $\Delta \mathrm{Ct}$ values of the two independent groups assessed in gene expression studies, although data may be represented as percentage or fold change in the figures. Statistical significance was achieved if $P<0.05$.

\section{Results}

CHD-FA has rapid and broad-spectrum antibacterial activity

Corsodyl $^{\odot}(0.2 \% \mathrm{CHX})$ and Dentracine (0.8\% CHD-FA) are oral formulations containing the active ingredients CHX and CHD-FA, respectively. Both agents were shown to be highly active against all planktonic and sessile oral bacteria tested (data not shown). The studies described in this manuscript focussed on the active ingredients $\mathrm{CHX}$ and CHD-FA and both were shown to be 
Table 1 Susceptibility profile of clinically relevant oral bacteria to four antimicrobial agents

\begin{tabular}{|c|c|c|c|c|c|c|c|c|}
\hline \multicolumn{9}{|c|}{ MIC (\%) } \\
\hline \multirow[b]{2}{*}{ Organism } & \multicolumn{4}{|c|}{ CHD-FA } & \multicolumn{4}{|c|}{$\mathrm{CHX}$} \\
\hline & PMIC & MBC & SMIC & $\begin{array}{l}\text { Fold change } \\
\text { (SMIC/PMIC) }\end{array}$ & PMIC & MBC & SMIC & $\begin{array}{l}\text { Fold change } \\
\text { (SMIC/PMIC) }\end{array}$ \\
\hline A. $a^{*}$ & 0.25 & 0.25 & 0.5 & 2 & 0.00078 & 0.00313 & 0.025 & 32 \\
\hline S. mitis & 0.0625 & 0.125 & 0.5 & 8 & 0.00078 & 0.00156 & 0.0125 & 16 \\
\hline S. mutans & 0.25 & 0.25 & 0.5 & 2 & 0.00039 & 0.00078 & 0.025 & 64 \\
\hline E. faecalis & 0.125 & 0.25 & 0.5 & 4 & 0.00156 & 0.025 & 0.003 & 2 \\
\hline F. nucleatum & 0.125 & 0.5 & 0.5 & 4 & 0.00039 & 0.00078 & 0.195 & 32 \\
\hline P. gingivalis & 0.0625 & 0.5 & 0.5 & 8 & $\leq 0.00039$ & 0.00078 & 0.0625 & $\geq 16$ \\
\hline
\end{tabular}

${ }^{*}$ A. actinomycetemcomitans.

CHD-FA - (Fulhold Ltd, South Africa), CHX - (GlaxoSmithKline Consumer Health Care, UK).

highly efficient at inhibiting and killing all oral isolates tested when grown either planktonically and as biofilms (Table 1). PMICs for the oral isolates ranged from $0.0625 \%$ to $0.25 \%$ for CHD-FA and from $<0.00039 \%$ to $0.00078 \%$ for CHX. The PMBC/PMIC ratio for CHD-FA and $\mathrm{CHX}$ were $\leq 4$, indicating both compounds displayed bactericidal activity. None of the bacterial species tested were notably more sensitive or resistant to either of the compounds. Both CHD-FA and CHX showed activity against mature biofilms, with SMICs of $0.5 \%$ for CHD-FA and from $0.003 \%$ to $0.025 \%$ for CHX. Interestingly, although CHX was effective at lower concentrations, the
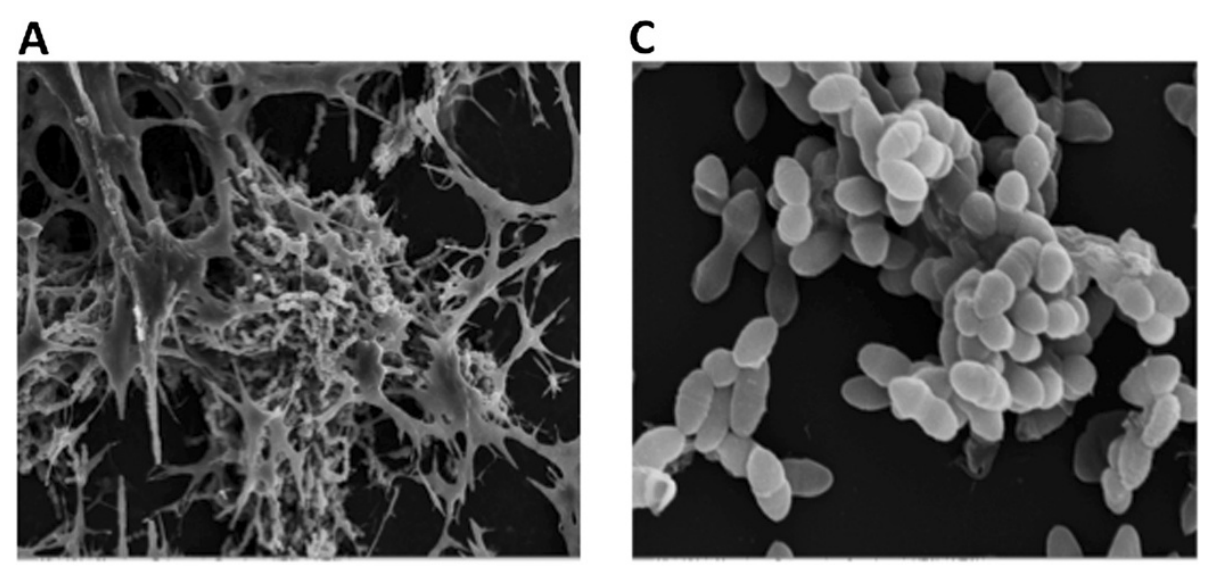

\section{B}

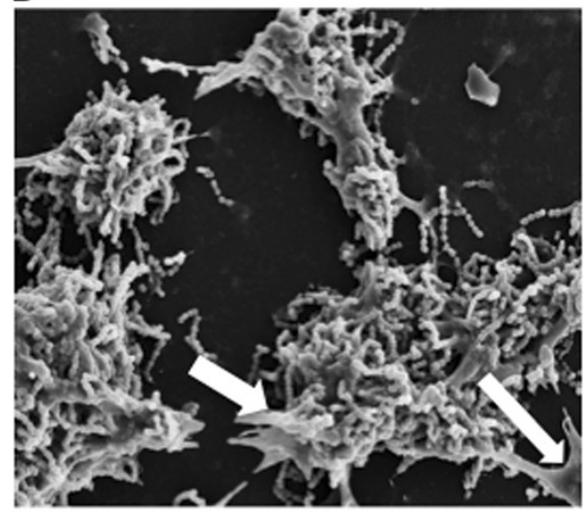

D

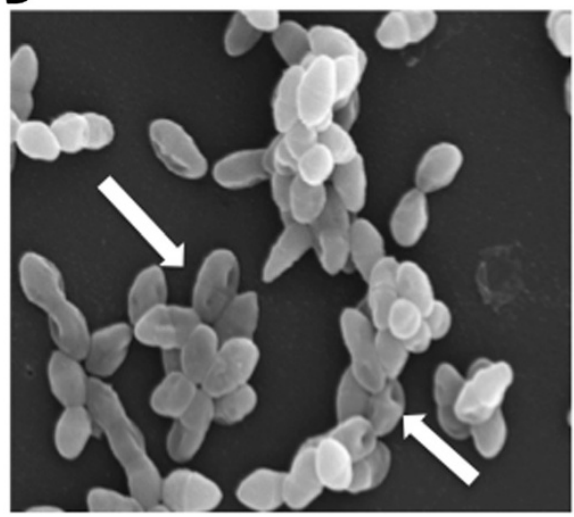

Figure 1 CHD-FA reduces ECM and compromises cell membrane structure. S. mutans (x2000) and E. faecalis ( $\times 5000)$ biofilms were either untreated ( $\mathbf{A}$ and $\mathbf{C}$, respectively) or treated with $0.5 \%(\mathrm{~V} / \mathrm{V})$ CHD-FA (B and $\mathbf{D}$, respectively) for $24 \mathrm{~h}$ on Thermanox ${ }^{\mathrm{TM}}$ coverslips. These were then processed and viewed on a JEOL JSM-6400 scanning electron microscope and images assembled using Photoshop software. Note the reduction in extracellular matrix (B) and perturbation of bacterial cell membranes (D), denoted by arrows on sessile cells. 


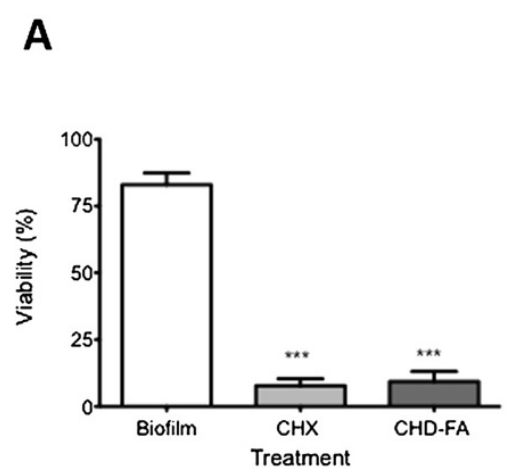

C

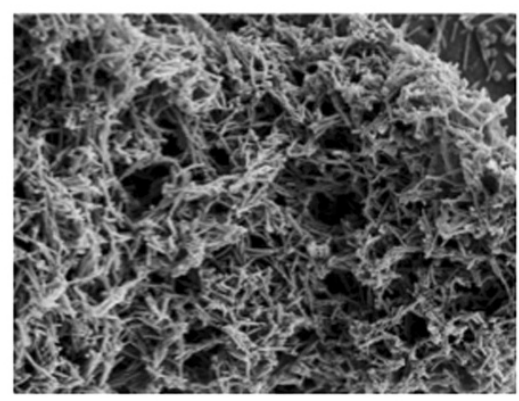

E



G



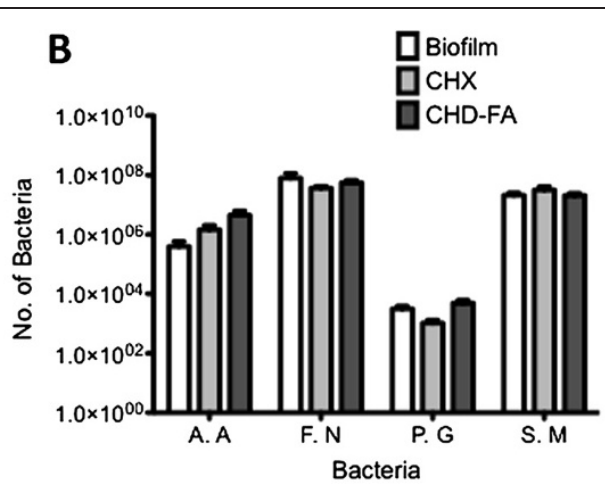

D

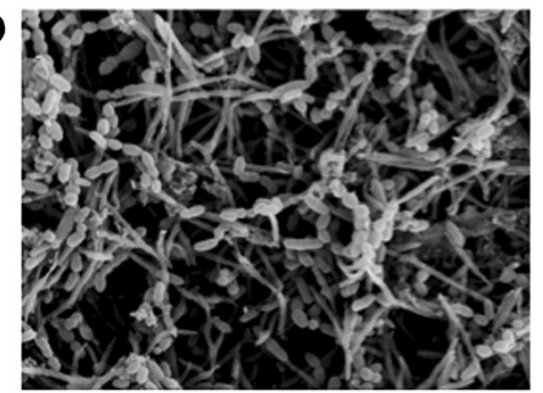

$\mathbf{F}$

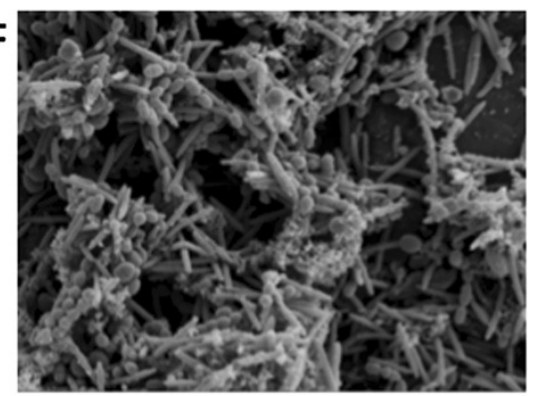

$\mathrm{H}$

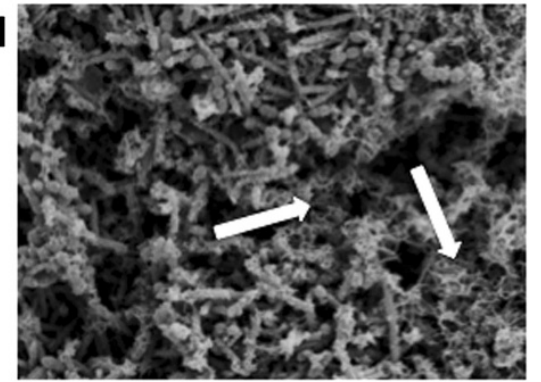

Figure 2 CHD-FA kills and disrupts multi-species periodontal biofilms. Multi-species periodontal biofilms were grown on Thermanox ${ }^{\mathrm{TM}}$ coverslips within 24 well plates for a total of 5 days, with AS media changed every day. Upon biofilm development, cells were treated with $0.5 \%$ $(\mathrm{v} / \mathrm{V})$ CHD-FA and $0.2 \%(\mathrm{v} / \mathrm{V}) \mathrm{CHX}$ for $24 \mathrm{~h}$ before being washed with PBS. Reduction in metabolic activity was measured using the alamarBlue ${ }^{\odot}$ assay (A). All samples were assayed in triplicate, on three separate occasions. Data represents mean \pm SD $(* * * p<0.0001)$. Biofilms were retained after treatment with CHD-FA or CHX and DNA was extracted for quantification of each species using SYBR ${ }^{\circledast}$ GreenER ${ }^{\text {TM }}$ based qPCR (B). Biofilms were also analysed by SEM at either 2000x (C, E, G) and 5000x (D, F, H). These were processed and viewed on a JEOL JSM-6400 scanning electron microscope and images assembled using Photoshop software. Untreated multispecies biofilms were first compared at low magnification (C) to biofilms treated with $0.2 \%(\mathrm{v} / \mathrm{V}) \mathrm{CHX}(\mathbf{E})$ and $0.5 \%(\mathrm{~V} / \mathrm{V})$ CHD-FA (G) for $24 \mathrm{~h}$ and it was shown that biofilm treatments caused disaggregation. At higher magnification the biofilms treated with $0.5 \%(\mathrm{~V} / \mathrm{V}) \mathrm{CHD}-\mathrm{FA}$ for $24 \mathrm{~h}$ resulted in a fibrous ECM, as denoted by arrows $(\mathbf{H})$, as compared to the control (D) and CHX (F).

fold change from PMIC to SMIC ranged from only 2 to 8 for CHD-FA, whereas for CHX this ranged from 2 to 64. Overall, $P$. gingivalis was the most susceptible organism to the antimicrobial therapies tested, particularly for planktonic cells. In addition, all bacterial biofilms were equally susceptible to CHD-FA with a SMIC of $0.5 \%$. 
Further analysis of the impact of CHD-FA upon the physical cellular structure was assessed by SEM for representative biofilms. CHD-FA treatment reduced the overall quantity of $S$. mutans ECM (Figure 1B) and also caused perturbation of the cell membrane, as demonstrated by the punctured appearance of E. faecalis (Figure 1D).

\section{CHD-FA is effective against a multi-species periodontal biofilm}

Given that biofilms of the oral cavity are polymicrobial in nature, we developed a simple and reproducible multispecies model representative of sub-gingival plaque to test CHD-FA (Figure 2A). The antimicrobial activity of CHDFA at $1 \times$ SMIC was shown to significantly reduce cell viability to less than $10 \%(\mathrm{p}<0.0001)$, which was comparable to the $\mathrm{CHX}$, which also significantly reduced cell viability to $8 \%(\mathrm{p}<0.0001)$. However, following treatment the number of each species within the biofilms were quantified and showed no significant reduction in biomass after CHD-FA or CHX treatment, compared to the untreated control (Figure 2B).

SEM analysis of these biofilms was then performed to evaluate any effect on the biofilm architecture (Figure 2C$\mathrm{H})$. At low magnification (x2000) both CHX and CHD-FA were shown to disrupt biofilm architecture (Figure 2E and G) when compared to the untreated control (Figure 2C), as shown by areas of sparse disaggregated biofilms. Moreover, at high magnification (x5000) CHD-FA also appeared to alter the overall physical appearance of the biofilm matrix with greater quantities of fibrous ECM observed as denoted by the arrows (Figure $2 \mathrm{H}$ ), compared to the control and CHX (Figure 2D and F).

CHD-FA alters the expression of pro-inflammatory mediators CHD-FA toxicity was assessed using an orally relevant epithelial cell line to determine whether there were any detrimental effects from the compounds tested prior to immunomodulatory investigations. Both $\mathrm{CHX}$ and $\mathrm{CHD}-$ $\mathrm{FA}$, at its native $\mathrm{pH}$ of 2.0 , were shown to be highly toxic towards to epithelial cells, reducing viability to less than $10 \%$ after $30 \mathrm{~min}$ exposure (Figure 3). However, when CHD-FA was buffered to a neutral $\mathrm{pH}$ of 7.0, no significant decrease in cell viability was observed.

We next set out to determine whether or not CHD-FA was able to induce a biological response from the epithelial cells, principally by measuring changes in immune mediators. To evaluate this, a four-species biofilm model was developed, where no toxicity issues were observed when in contact with the epithelial cell line at $4 \mathrm{~h}$ (data not shown). Moreover, we have shown that CHD-FA treated cells do not significantly alter the release of IL- 8 after $4 \mathrm{~h}$ and $24 \mathrm{~h}$ (data not shown). Initial gene expression studies using the $\mathrm{RT}^{2}$ Profiler on epithelial cells pre-treated with CHD-FA prior to biofilm challenge showed a general down-

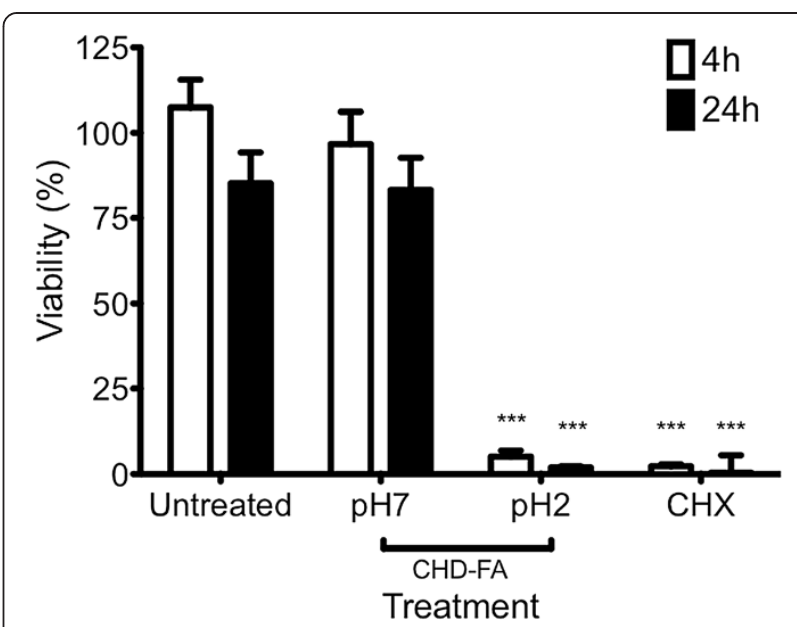

Figure 3 CHD-FA is non-toxic against an oral epithelial cell line. An orally relevant epithelial cell line (OKF6/TERT2) was grown to 90\% confluence in 24 well plates for toxicity studies. Cells were treated with $0.5 \%$ (v/v) CHD-FA at pH 2.0 and 7.0 and with $0.2 \%$ CHX for 30 min. After treatment, cells were carefully washed with PBS and cellular viability assessed using the alamarBlue ${ }^{\circledR}$ assay, with absorbance read at 570 and $600 \mathrm{~nm}$. All samples were assayed in triplicate, on three independent occasions. Data represents mean \pm SD $\left({ }^{* *} p<0.0001\right)$.

regulation of pro-inflammatory mediators (Figure 4A). Significant down-regulated genes included IL-6 (8.5 fold $[\mathrm{p}=0.018]), \mathrm{IL}-1 \beta(7.05$ fold $[\mathrm{p}=0.012]), \mathrm{TNF} \alpha(5.22$ fold $[\mathrm{p}=0.013])$ and IL-8 (4.24 fold [p = 0.021]).

We next focussed on IL-8 expression, one of the significantly affected genes and a key mediator of periodontal inflammation. At the mRNA level, IL-8 was significantly down-regulated in cells pre-treated with CHD-FA after $4 \mathrm{~h}$, when compared to the untreated control $(p=0.0383)$ (Figure 4B). No statistically significant difference was observed after $24 \mathrm{~h}$ stimulation $(\mathrm{p}=0.1712)$. In addition, at the protein level, IL- 8 release was shown to be significantly down-regulated when cells were pre-treated with CHD-FA, after $4 \mathrm{~h}(\mathrm{p}=0.008)$ and $24 \mathrm{~h}$ $(\mathrm{p}=0.0037)$ biofilm stimulation (Figure 4C). CHD-FA alone had no effect on oral epithelial cell IL-8 mRNA or protein expression (data not shown).

\section{Discussion}

Oral microbial diseases are typically mediated by biofilms; communities of microorganisms that co-aggregate as sticky and tenacious structures and which characteristically have increased resistance to antimicrobials [25]. We recently reported that mouthwashes, including $\mathrm{CHX}$ were ineffective against a range of clinical MRSA strains [10], suggesting that alternative antimicrobial agents ought to be investigated. Indeed, recent studies in C. albicans have shown that the use of naturally derived molecules are effective against both orally and systemically derived isolates $[14,15]$. Here we report that CHD-FA, a naturally derived 
Figure 4 CHD-FA modulates key inflammatory mediators in vitro. An oral epithelial cell line (OKF6) was grown to 90\% confluence in 24 well plates for assessing the effect of CHD-FA on the host immune response. Cells were pre-treated with $0.5 \%(\mathrm{v} / \mathrm{v})$ CHD-FA pH 7.0 for 30 min, washed with PBS and stimulated with the multi-species periodontal biofilm for 4 and $24 \mathrm{~h}$. Untreated controls were also included. Samples were assayed in triplicate and on three separate occasions. RNA was extracted from the $4 \mathrm{~h}$ cell lysates, CDNA synthesised and used in the $\mathrm{RT}^{2}$ Profiler to analyse the expression of a panel of pro-inflammatory mediators (A). Duplicate samples from two independent experiments were used in the $\mathrm{RT}^{2}$ Profiler. Data is represented by mean $+95 \% \mathrm{Cl}$, relative to untreated control. Samples were also assayed for IL-8 gene expression using $\mathrm{SYBR}^{\oplus}$ GreenER ${ }^{\mathrm{TM}}$ based $\mathrm{qPCR}$ (B). Expression of IL-8 is represented relative to housekeeping gene; GAPDH. Retained supernatants were also used to measure IL-8 protein release by ELISA (C). All samples were assayed in triplicate, on three independent occasions. Data represents mean $\pm S D\left({ }^{*} p<0.05,{ }^{* *} p<0.01\right)$.

antiseptic molecule, displays rapid broad-spectrum antimicrobial activity, and also elicits immunomodulatory activity.

We first undertook a comparative assessment of CHDFA and CHX against a range of important bacteria associated with oral biofilm infections. Both molecules were shown to effectively inhibit and kill planktonic cells, and both compounds were also effective against biofilms. Antimicrobial activity against planktonic bacteria has been reported previously for oxifulvic acid, a derivative of CHD-FA, where inhibition was observed against a range of important clinical pathogens, including Pseudomonas aeruginosa, Staphylococcus aureus and Streptococcus pyogenes [26]. Notably, only marginally higher concentrations of CHD-FA were required to kill the biofilm as compared to planktonic cells, whereas for $\mathrm{CHX}$ the fold change was up to 64 times, a phenomenon also reported for CHD-FA against C. albicans biofilms [15]. Moreover, CHD-FA showed a rapid rate of kill for the periodontal pathogens tested as polymicrobial biofilms, as after $30 \mathrm{~min}$ treatment cellular viability was reduced by $\geq 90 \%$, which was also observed for studies of $C$. albicans [15]. It is recognised that a potential limitation of this study is that it was performed on biofilms produced from laboratory strains of the periodontal organisms in vitro and it is conceded that further investigation may be required to assess the anti-microbial properties against the most virulent of clinical strains and biofilms formed from ex vivo biofilms or in vivo within experimental gingivitis models. Nevertheless, collectively, these data suggest that CHD-FA has potent and broad-spectrum activity against microbial biofilms. Furthermore, the SEM images indicate an action against the bacterial cell membrane. Interestingly, the biofilms appeared to be disaggregated and displayed a fibrous appearance, presumably as a consequence of cell lysis and release of intracellular components of the bacterial cells. Our previous studies on C. albicans biofilms do 
not corroborate this observation, where no disruption of biofilms was observed [15]. However, the filamentous nature of $C$. albicans biofilms may explain why the compound was unable to disaggregate these. Despite this finding, there was no significant difference in the number of each species when treated with antimicrobial therapy, when compared to the untreated control. Though our assay was unable to determine whether these were live or dead.

Given that CHD-FA displayed an excellent antimicrobial profile, we wanted to ascertain whether it possesses any other biological properties, as has also been demonstrated for other naturals including tea tree oil [27]. It has been reported that CHD-FA has no toxicity in rats and humans, and it has been further suggested that it elicits anti-inflammatory and wound healing promoting properties $[17,28]$. Periodontitis is characterised by chronic inflammation that leads to tissue and bone destruction [29], therefore controlling these processes is an attractive option for clinical management. The use of in vitro multi-species oral biofilms to study the inflammatory processes driven by complex biofilms have been shown to be important [30], therefore we developed a similar system to test CHD-FA and other bioactive molecules. Both CHX and CHD-FA in their native forms were shown to be toxic, therefore, in order to demonstrate subtle biological effects we buffered CHD-FA to $\mathrm{pH} 7.0$ in order to test our hypothesis that it was immunomodulatory in vitro. Using an orally relevant epithelial cell line stimulated with a polymicrobial biofilm we demonstrated that at the transcript level cells treated with CHD-FA showed a significant down-regulation of pro-inflammatory molecules, including the chemokine IL-8. Analysis of the IL-8 protein also showed a significant reduction in its release from oral epithelial cells. These data indicate that CHD-FA has bioactivity against mammalian cells, as has been reported elsewhere $[17,28]$. However, we accept these differences are only observed when CHD-FA is adjusted to a neutral $\mathrm{pH}$, therefore, further studies are required to determine the most suitable formulation of CHD-FA to potentially be used clinically, which at present is formulated at $\mathrm{pH} 2.8$. At present, however, the precise mechanism of action remains unknown, but we can only speculate that CHDFA interacts with membrane proteins resulting in blocking signalling pathways, which leads to downregulation of pro-inflammatory mediators on stimulation with biofilms. This is currently subject to further investigation by our group.

\section{Conclusions}

This study has demonstrated that the naturally derived compound CHD-FA exhibits broad-spectrum antimicrobial activity against orally relevant biofilm organisms.
Although a four species mixed biofilm model was used in this study, we are aware that antimicrobial activity against this model does not fully represent all mixed biofilms that are found within the oral cavity, but only a few of species relevant in periodontal disease. It further shows that CHD-FA has the capacity to modulate the immune response and down-regulate the biofilm induced expression of pro-inflammatory mediators in oral keratinocytes. However, a further limitation of this study was only a selected number of inflammatory mediators were investigated, thus precluding other host factors for consideration, which may influence the inflammatory response even further. Collectively, these properties make CHD-FA an attractive option for the development of a mouthwash to treat microbial oral disease; although further studies in vitro and in vivo are first required to further define the mode of action of this unique compound.

\section{Abbreviations}

CHD-FA: Carbohydrate derived fulvic acid; ECM: Extracellular matrix; CHX: Chlorhexidine; SEM: Scanning electron microscopy; Cl: Confidence intervals; SD: Standard deviation; PMIC: Planktonic minimum inhibitory concentration; PMBC: Planktonic minimum bactericidal concentration; SMIC: Sessile minimum inhibitory concentration; TTO: Tea tree oil; MRSA: Methicillin resistance Staphylococcus aureus.

\section{Competing interests}

The PhD studentship stipend of LS was partially funded by Fulhold Ltd, who also provided CHD-FA for the experimental procedures.

\section{Authors' contributions}

LS participated in the study design, carried out the experimental studies, performed statistical analysis and was responsible for the manuscript. EM produced and ran the multi-species biofilm model. DFL participated in study design, assisted with statistical support and helped draft the manuscript. CM participated in study design and supervised manuscript writing. SC contributed to study design, data analysis and supervised manuscript writing. CJN contributed to the immunological study design, data analysis and contributed to the manuscript writing. GR conceived the study, participated in study design and was jointly responsible for writing the final manuscript. All authors read and approved the manuscript.

\section{Acknowledgements}

Margaret Mullin (University of Glasgow) for her assistance in scanning electron microscopy techniques.

Received: 11 June 2013 Accepted: 23 September 2013

Published: 24 September 2013

\section{References}

1. Peyyala R, Ebersole $\mathrm{J}$ : Multispecies biofilms and host responses: "discriminating the trees from the forest". Cytokine 2013, 61(1):15-25.

2. Yamakami K, Tsumori H, Sakurai Y, Shimizu Y, Nagatoshi K, Sonomoto K: Sustainable inhibition efficacy of liposome-encapsulated nisin on insoluble glucan-biofilm synthesis by streptococcus mutans. Pharm Biol 2012, 51(2):267-270.

3. Ramage G, Rajendran R, Sherry L, Williams C: Fungal biofilm resistance. Int J Microbiol 2012, 2012:528521.

4. Soto SM: Role of efflux pumps in the antibiotic resistance of bacteria embedded in a biofilm. Virulence 2013, 4(3):223-229.

5. Herrera D: Chlorhexidine mouthwash reduces plaque and gingivitis. Evid Based Dent 2013, 14(1):17-18.

6. Najafi MH, Taheri M, Mokhtari MR, Forouzanfar A, Farazi F, Mirzaee M, Ebrahiminik Z, Mehrara R: Comparative study of $0.2 \%$ and $0.12 \%$ digluconate chlorhexidine mouth rinses on the level of dental staining and gingival indices. Dental research journal 2012, 9(3):305-308. 
7. Quirynen M, Avontroodt P, Peeters W, Pauwels M, Coucke W, van Steenberghe D: Effect of different chlorhexidine formulations in mouthrinses on de novo plaque formation. J Clin Periodontol 2001, 28(12):1127-1136.

8. Jones CG: Chlorhexidine: is it still the gold standard? Periodontol 2000 1997, 15:55-62.

9. Pemberton MN, Gibson J: Chlorhexidine and hypersensitivity reactions in dentistry. Br Dent J 2012, 213(11):547-550.

10. Smith K, Robertson DP, Lappin DF, Ramage G: Commercial mouthwashes are ineffective against oral MRSA biofilms. Oral Surg Oral Med Oral Pathol Oral Radiol 2013, 115(5):624-629.

11. Turkoglu O, Becerik S, Emingil G, Kutukculer N, Baylas H, Atilla G: The effect of adjunctive chlorhexidine mouthrinse on clinical parameters and gingival crevicular fluid cytokine levels in untreated plaque-associated gingivitis. Inflamm Res 2009, 58(5):277-283.

12. Liu $Y$, Zhang $Y$, Wang $L, G$ Go $Y$, Xiao S: Prevalence of porphyromonas gingivalis four rag locus genotypes in patients of orthodontic gingivitis and periodontitis. PloS one 2013, 8(4):e61028.

13. Houri-Haddad $Y$, Halabi A, Soskolne WA: Inflammatory response to chlorhexidine, minocycline $\mathrm{HCl}$ and doxycycline $\mathrm{HCl}$ in an in vivo mouse model. J Clin Periodontol 2008, 35(9):783-788.

14. Ramage G, Milligan S, Lappin DF, Sherry L, Sweeney P, Williams C, Bagg J, Culshaw S: Antifungal, cytotoxic, and immunomodulatory properties of tea tree oil and its derivative components: potential role in management of oral candidosis in cancer patients. Front Microbiol 2012, 3:220.

15. Sherry L, Jose A, Murray C, Williams C, Jones B, Millington O, Bagg J, Ramage G: Carbohydrate derived fulvic acid: an in vitro investigation of a novel membrane active antiseptic agent against Candida albicans biofilms. Front Microbiol 2012, 3:116.

16. Ahmad A, Khan A, Kumar P, Bhatt RP, Manzoor N: Antifungal activity of coriaria nepalensis essential oil by disrupting ergosterol biosynthesis and membrane integrity against Candida. Yeast 2011, 28(8):611-617.

17. Gandy JJ, Snyman JR, van Rensburg CE: Randomized, parallel-group, double-blind, controlled study to evaluate the efficacy and safety of carbohydrate-derived fulvic acid in topical treatment of eczema. Clin Cosmet Investig Dermatol 2011, 4:145-148.

18. CLSI: Methods for antimicrobial susceptibility testing of anaerobic bacteria; approved standard - eighth edition. CLSI document M11-A8, Wayne, PA: CLSI 2012, 27(2).

19. CLSI: Methods for dilution antimicrobial susceptibility test for bacteria that grow aerobically; approved standard - nineth edition. CLSI document M7-A9, Wayne, PA: CLSI 2011, 26(2).

20. Pierce CG, Uppuluri P, Tristan AR, Wormley FL Jr, Mowat E, Ramage G, Lopez-Ribot JL: A simple and reproducible 96 -well plate-based method for the formation of fungal biofilms and its application to antifungal susceptibility testing. Nat Protoc 2008, 3(9):1494-1500.

21. Kirchner S, Fothergill JL, Wright EA, James CE, Mowat E, Winstanley C: Use of artificial sputum medium to test antibiotic efficacy against pseudomonas aeruginosa in conditions more relevant to the cystic fibrosis lung. Journal of Visualized Experiments: JOVE 2012, 64:e3857.

22. Pratten J, Smith AW, Wilson M: Response of single species biofilms and microcosm dental plaques to pulsing with chlorhexidine. J Antimicrob Chemother 1998, 42(4):453-459.

23. Erlandsen SL, Kristich CJ, Dunny GM, Wells CL: High-resolution visualization of the microbial glycocalyx with low-voltage scanning electron microscopy: dependence on cationic dyes. J Histochem Cytochem 2004 52(11):1427-1435.

24. Livak KJ, Schmittgen TD: Analysis of relative gene expression data using real-time quantitative $P C R$ and the $2(-$ delta delta $C(T))$ method. Methods 2001, 25(4):402-408.

25. ten Cate JM, Zaura E: The numerous microbial species in oral biofilms: how could antibacterial therapy be effective? Adv Dent Res 2012, 24(2):108-111.

26. van Rensburg CE, van Straten A, Dekker J: An in vitro investigation of the antimicrobial activity of oxifulvic acid. $J$ Antimicrob Chemother 2000, 46(5):853.

27. Carson CF, Hammer KA, Riley TV: Melaleuca alternifolia (Tea tree) oil: a review of antimicrobial and other medicinal properties. Clin Microbiol Rev 2006, 19(1):50-62.
28. Sabi R, Vrey P, van Rensburg CE: Carbohydrate-derived fulvic acid (CHD-FA) inhibits carrageenan-induced inflammation and enhances wound healing: efficacy and toxicity study in rats. Drug Dev Res 2011, 73(1):18-23.

29. Berker E, Kantarci A, Hasturk H, Van Dyke TE: Blocking Pro-inflammatory cytokine release modulates peripheral blood mononuclear cell response to porphyromonas gingivalis. J Periodontol 2012, 84(9):1337-1345.

30. Peyyala R, Kirakodu SS, Novak KF, Ebersole JL: Oral epithelial cell responses to multispecies microbial biofilms. J Dent Res 2013, 92(3):235-240.

\section{doi:10.1186/1472-6831-13-47}

Cite this article as: Sherry et al:: Investigating the biological properties of carbohydrate derived fulvic acid (CHD-FA) as a potential novel therapy for the management of oral biofilm infections. BMC Oral Health 2013 13:47.

\section{Submit your next manuscript to BioMed Central and take full advantage of:}

- Convenient online submission

- Thorough peer review

- No space constraints or color figure charges

- Immediate publication on acceptance

- Inclusion in PubMed, CAS, Scopus and Google Scholar

- Research which is freely available for redistribution 